\title{
Third molar surgical difficulty scales: systematic review and preoperative assessment form
}

\author{
Cosme Gay-Escoda ${ }^{1}$, Alba Sánchez-Torres ${ }^{2}$, Jordi Borrás-Ferreres ${ }^{3}$, Eduard Valmaseda-Castellón ${ }^{4}$
}

${ }^{1} \mathrm{MD}$, DDS, MS, PhD, EBOS, OMFS. Chairman and Professor of the Oral and Maxillofacial Surgery Department, School of Medicine and Health Sciences, University of Barcelona. Director of Master's Degree Program in Oral Surgery and Implantology (EFHRE International University / FUCSO). Coordinator and Researcher of the IDIBELL Institute. Head of Oral and Maxillofacial Surgery and Implantology Department of the Teknon Medical Centre, Barcelona, Spain

${ }^{2}$ DDS, MS, Master of Oral Surgery and Implantology. Associate Professor of Oral Surgery, School of Medicine and Health Sciences, University of Barcelona. Researcher at the IDIBELL Institute, Barcelona, Spain

${ }^{3}$ DDS. Professor of the Master's Degree Program in Oral Surgery and Implantology, EFHRE International University/FUCSO. Postgraduate degree on Temporomandibular Disorders and Orofacial Pain, SCOE, Barcelona, Spain

${ }^{4}$ DDS, MS, PhD, EBOS. Professor of Oral Surgery and Director of the Master's degree program in Oral Surgery and Implantology, School of Medicine and Health Sciences, University of Barcelona. Researcher at the IDIBELL Institute, Spain

Correspondence:

School of Medicine and Health Sciences

Campus de Bellvitge. University of Barcelona

C/ Feixa Llarga, s/n; Pavelló Govern, $2^{a}$ planta, Despatx 2.9

08907, L'Hospitalet de Llobregat, Barcelona, Spain

albaschez@gmail.com

Received: 23/06/202

Accepted: 04/11/202

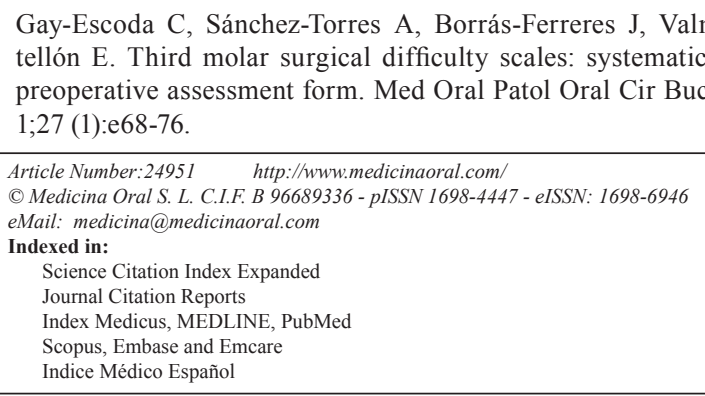

\begin{abstract}
Background: The main objective of this systematic review was to collect the pre-existing scales for assessing the difficulty of third molar extraction. The secondary objective was to design a proposal for a preoperative evaluation protocol for the difficulty of third molar extraction.

Material and Methods: Two independent researchers conducted an electronic search in Pubmed (MEDLINE), Cochrane, and Scopus databases during March 2021. Included studies evaluated the prediction of the difficulty of surgical removal of impacted upper or lower third molars using new indices/scales or pre-existing scales with or without modifications. Articles referring to coronectomies or assessing pre-surgical difficulty using other tools were excluded. Neither language nor publication date restrictions were applied.

Results: Out of 242 articles, 13 prospective cohort studies were finally selected. Seven developed new indices/ scales, and 6 assessed the predictive ability of some pre-existing scales. Most of the indices/scales contained radiological variables and few added any patient-related variables. We proposed a preoperative assessment protocol of the difficulty of third molar extraction to facilitate treatment planning and/or considerate referral in cases of high difficulty. This proposal used patient-related, radiological and surgical variables.

Conclusions: Using a preoperative protocol to evaluate the surgical difficulty, including different patient-specific, radiological and surgical variables, could facilitate treatment planning, help clinicians prevent complications and assess the possibility of referral.
\end{abstract}

Key words: Wisdom teeth, patient characteristics, radiological variables, surgeon experience, assessment form. 


\section{Introduction}

Removal of third molars (3M) is one of the most common procedures in oral surgery. Pre-operative evaluation of surgical difficulty can help the practitioner plan the surgical technique, estimate the operating time and foresee possible complications (1). In addition, the practitioner can also evaluate the ability to perform the surgery or, if more appropriate, refer the patient to a more qualified oral surgery specialist (2). Renton et al. (3) underlined the relevance of preoperative assessment of the surgical difficulty of $3 \mathrm{Ms}$ from a teaching point of view, since dental or radiological factors are usually more considered in preoperative training, though expert surgeons usually assess other clinical or demographic variables. The ability to predict surgical difficulty based on the surgeon's experience is controversial, as in the published literature some studies find no difference (4), while others have even observed a trend towards better estimation of difficulty for each year of training and high values for experienced maxillofacial surgeons (5). The fact that most $3 \mathrm{M}$ difficulty scales are mainly based on radiological criteria constitutes a gap between the impact that patient or surgeon factors can have on actual surgical difficulty (6). In this regard, the American Association of Endodontists has developed an assessment form called 'Endodontic Case Difficulty Assessment Form and Guidelines' to be used in endodontic curricula as a guide for teachers to assist students in making a correct decision process.

In the field of oral surgery there is no form to determine the difficulty and assess the ability to perform surgery or to refer the case to a specialist according to the different variables involved, such as patient, radiological and operative factors, as determined by a recently published systematic review (6). Considering that diagnosis of third molars is usually performed in primary care services, a tool to assess the difficulty of third molar extractions could help both general dental practitioners and more experienced surgeons select the proper setting for third molar extractions.

The main objective of this systematic review was to collect the pre-existing scales to assess the difficulty of $3 \mathrm{M}$ extraction. The secondary objective was to design a $3 \mathrm{M}$ difficulty assessment form, based on the previously demonstrated influencing factors, to assist clinicians, whether they are students, recent graduates or even oral surgery specialists, to make a correct treatment plan or to make a referral decision.

\section{Material and Methods}

This systematic review was carried out according to Preferred Reporting Items for Systematic Reviews and Meta-Analyses (PRISMA) guidelines (7) and the review protocol was registered in PROSPERO database (number CRD42020186643).
Inclusion criteria were studies assessing the preoperative prediction of the difficulty of impacted upper or lower 3M removal using new indices/scales or preexisting scales with or without modifications. Articles referring to coronectomies or that had only evaluated the preoperative difficulty by means of visual analogue scales or operating time were excluded. Neither language nor publication date restrictions were applied. Two independent researchers (AST, JBF) performed an electronic search in Pubmed (MEDLINE), Cochrane, and Scopus databases during March 2021. The search strategy used was "(wisdom tooth OR third molar) AND (scale difficulty OR difficulty guideline OR difficulty form OR difficulty classification OR difficulty index)". Articles were first selected by reading titles and abstracts, and finally, those that met the eligibility criteria were read in full text. A third researcher resolved any discrepancies (CGE). Moreover, a manual search into the references of the selected studies was also conducted to ensure that all studies related to the area of interest were collected. We calculated the degree of agreement between the researchers for article selection after the full text reading using Cohen's Kappa index.

Data was recorded in tables to collect the following information: author and year, number of patients and third molars treated, objective of the use of a scale or index (development of a new one or evaluation of a pre-existing one), name of the index or scale, type of variables (patient, radiological or surgical) and individual items recorded by the index/scale, objective post-operative variables that help determine the difficulty, evaluator of the index/scale, surgeon(s) experience and main results. Based on this information and the factors that determine an increase in difficulty according to a previous systematic review (7), the authors designed a guide for assessing the surgical difficulty of $3 \mathrm{M}$ removal. The level of evidence from the included articles was scored according to the Scottish Intercollegiate Guidelines Network (SIGN) grading system (8).

\section{Results}

The electronic search yielded 242 articles, of which 20 were selected to be read in full text. After reading, 7 articles were excluded because they did not assess the predictive ability of the indexes/scales (9-15). Finally, 13 articles were included in the systematic review (1628). All of them were prospective cohort studies and 1 was a multicenter study conducted in 3 centers (26). All of them assessed the difficulty of the $3 \mathrm{M}$ removal. Fig. 1 shows the flowchart of selected items according to PRISMA guidelines. The kappa index adjusted for bias and prevalence was 0.71 , which indicated substantial agreement between researchers for article selection. 

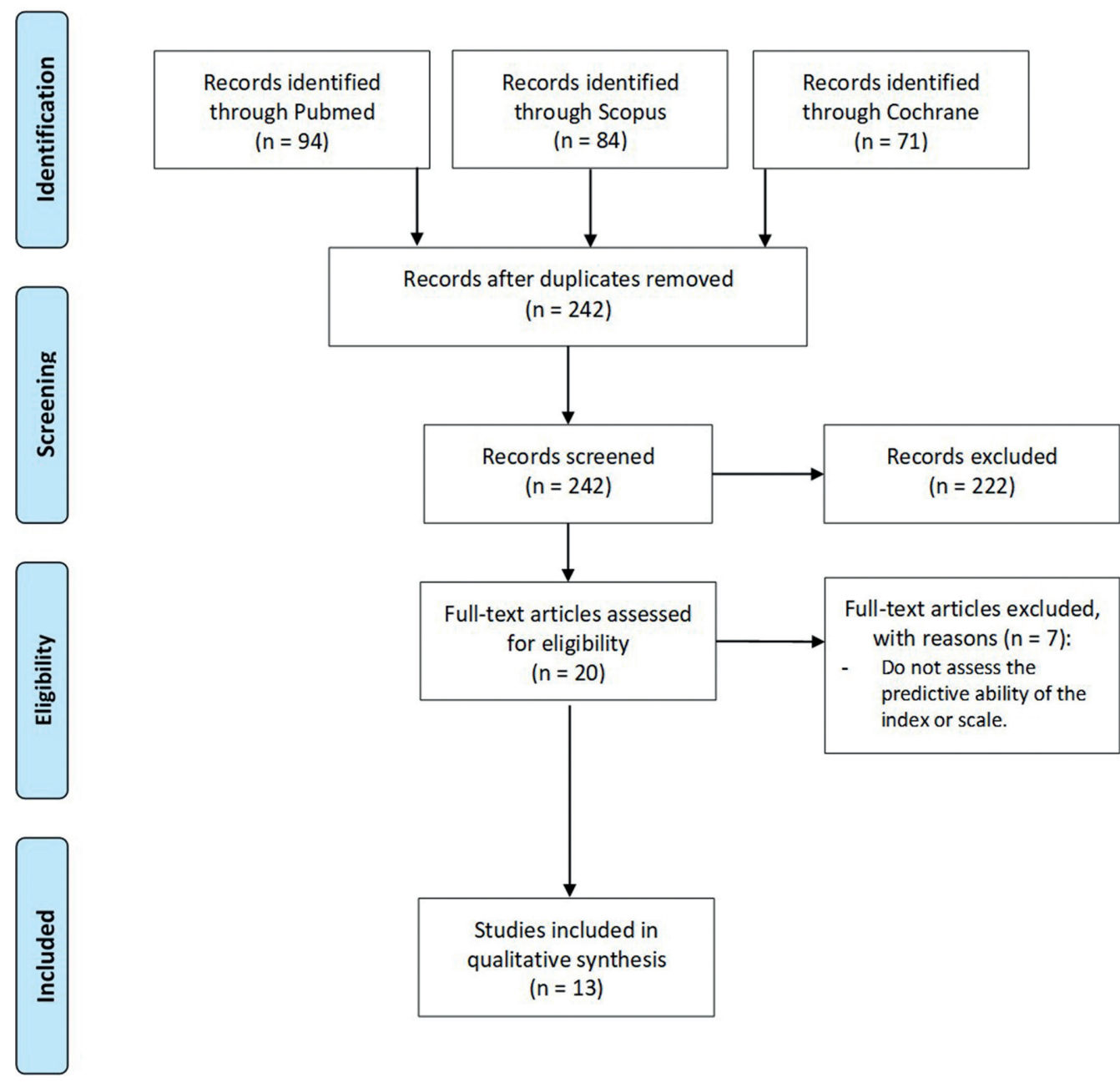

Fig. 1: Flow-chart of the selected articles throughout the systematic review process according to PRISMA statement.

Table 1 shows the main characteristics of the included studies. Six developed new indices/scales $(17,21,23-$ $25,27)$ and 5 assessed the predictive ability of preexisting indices/scales $(16,18-20,22,26,28)$. The most widely used pre-existing index/scale, both to assess its predictability and to compare it with new indices, was Pederson scale, which includes only the radiological variables of depth, available distal space and $3 \mathrm{M}$ angulation. In fact, the only studies that developed indices that add variables specific to patient characteristics were those published by Roy et al. (21), de Carvalho and Vasconcelos (25) and Zhang et al. (27). Age, body mass index (BMI), mouth opening, tongue size, angle of the external oblique ridge and cheek flex- ibility constitute the total of patient characteristics included in these indices/scales. The rest of indices/ scales evaluated included only radiological variables. Experience of the surgeon was not included in any of the indices/scales.

Most of the studies used the operative time (measured from the incision to the last suture) as a post-operative variable indicating the degree of difficulty $(17,18-21,24$ 28). Others used scales that evaluate the type of surgical technique $(16,18,20,24,25)$ and only 1 registered a score reported by the surgeon after the surgery to subjectively classify difficulty (23). Few studies reported on the experience of the surgeon(s) operating the cases included in the studies. 
Table 1: Main characteristics of the studies included.

\begin{tabular}{|c|c|c|c|c|c|c|c|c|c|c|}
\hline Author & \begin{tabular}{|l|} 
N pa- \\
tients \\
/teeth
\end{tabular} & Objective & $\begin{array}{l}\text { Index / } \\
\text { Scale }\end{array}$ & $\begin{array}{c}\text { Type of } \\
\text { varia- } \\
\text { bles }\end{array}$ & Items & \begin{tabular}{|c|} 
Post-operative \\
objective variable
\end{tabular} & $\begin{array}{l}\text { Evaluator } \\
\text { of index / } \\
\text { scale }\end{array}$ & \begin{tabular}{|c|}
$\begin{array}{c}\text { Surgeon } \\
\text { experien- } \\
\text { ce }\end{array}$ \\
\end{tabular} & Outcomes & $\begin{array}{l}\text { SIGN } \\
\text { score }\end{array}$ \\
\hline $\begin{array}{c}\text { Akadiri } \\
\text { et al. } \\
2009(22)\end{array}$ & $\begin{array}{l}79 / \\
79\end{array}$ & $\begin{array}{l}\text { Pre-exis- } \\
\text { tent index } \\
\text { / scale } \\
\text { evaluation }\end{array}$ & Pederson & \begin{tabular}{|l|} 
Radio- \\
logical
\end{tabular} & $\begin{array}{c}\text { Depth } \\
\text { Available distal space } \\
\text { Angulation }\end{array}$ & Operative time & \multicolumn{2}{|c|}{$\begin{array}{l}\text { A 9-year experienced } \\
\text { oral and maxillofacial } \\
\text { surgeon and a less } \\
\text { experienced surgeon }\end{array}$} & \begin{tabular}{|c|}
$\mathrm{Se}=94.9 \%$ \\
$\mathrm{Sp}=45 \%$ \\
$\mathrm{PPV}=62.7 \%$ \\
$\mathrm{NPV}=90 \%$ \\
Accuracy $=$ \\
$69.6 \%$
\end{tabular} & $2+$ \\
\hline $\begin{array}{c}\text { Al- } \\
\text { Samman } \\
2017(27)\end{array}$ & $\begin{array}{c}49 / \\
49\end{array}$ & $\begin{array}{l}\text { New index } \\
\text { / scale } \\
\text { develop- } \\
\text { ment }\end{array}$ & $\begin{array}{c}\text { Kharma } \\
\text { scale }\end{array}$ & \begin{tabular}{|l|} 
Radio- \\
logical
\end{tabular} & $\begin{array}{c}\text { Depth } \\
\text { Available distal space } \\
\text { Angulation } \\
\text { Root morphology }\end{array}$ & $\begin{array}{l}\frac{\text { Modified Parant }}{\text { scale }} \\
\text { I: Forceps } \\
\text { II: Ostectomy } \\
\text { III: Ostectomy and } \\
\text { odontosection } \\
\text { IV: Complex ex- } \\
\text { traction with root } \\
\text { sectioning } \\
\text { Operative time }\end{array}$ & \multicolumn{2}{|c|}{$\begin{array}{l}2 \text { surgeons specialized } \\
\text { in oral and maxilofa- } \\
\text { cial surgery with } 6 \text { and } \\
9 \text { years of experience }\end{array}$} & 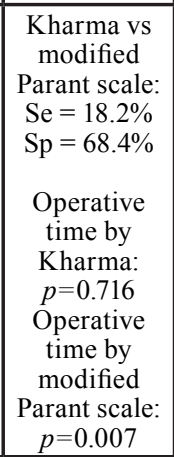 & $2+$ \\
\hline $\begin{array}{l}\text { Barrei- } \\
\text { ro-To- } \\
\text { rres et } \\
\text { al. 2010 } \\
(23)\end{array}$ & $\begin{array}{c}66 / \\
80\end{array}$ & $\begin{array}{l}\text { Pre-exis- } \\
\text { tent index } \\
\text { / scale } \\
\text { evaluation }\end{array}$ & \begin{tabular}{|c|} 
Modified \\
Parant \\
scale* \\
\\
Preop- \\
erative \\
VAS \\
$0-100$ \\
mm \\
\end{tabular} & - & - & $\begin{array}{l}\text { Modified Parant } \\
\text { scale* } \\
\text { Operative time }\end{array}$ & \begin{tabular}{|c|}
14 dentists: \\
2 from pri- \\
mary care \\
10 residents \\
of oral \\
surgery \\
2 maxi- \\
llofacial \\
surgeons \\
\end{tabular} & \begin{tabular}{|} 
Fellows \\
of Master \\
of Oral \\
Surgery
\end{tabular} & \begin{tabular}{|c|} 
Predictive \\
ability: \\
Primary care: \\
$31.9 \%$ \\
Residents: \\
$45.1 \%$ \\
Maxillofacial \\
surgeons: \\
$38.7 \%$ \\
\end{tabular} & $2+$ \\
\hline $\begin{array}{l}\text { Conti } \text { et } \\
\text { al. 2015 } \\
\text { (25) }\end{array}$ & $\begin{array}{c}1000 \\
/-\end{array}$ & $\begin{array}{l}\text { New index } \\
\text { / scale } \\
\text { develop- } \\
\text { ment }\end{array}$ & $\begin{array}{l}\text { Conti } \\
\text { scale }\end{array}$ & \begin{tabular}{|l|} 
Radio- \\
logical \\
Patient
\end{tabular} & $\begin{array}{c}\text { Tooth position and } \\
\text { orientation } \\
\text { Root morphology } \\
\text { Available distal space } \\
\text { Ankylosis } \\
\text { Patient general status } \\
\text { and attitude } \\
\text { Age } \\
\text { Mouth opening } \\
\text { Second molar rela- } \\
\text { tionship } \\
\text { Distance to mandibu- } \\
\text { lar canal } \\
\text { Residual bone volume }\end{array}$ & - & - & - & - & $2-$ \\
\hline $\begin{array}{l}\text { de Car- } \\
\text { valho } \\
\text { \& Vas- } \\
\text { concelos } \\
2018(28)\end{array}$ & $\begin{array}{l}-1 \\
280\end{array}$ & $\begin{array}{l}\text { New index } \\
\text { / scale de- } \\
\text { velopment }\end{array}$ & \begin{tabular}{|c|} 
Pernam- \\
buco \\
index
\end{tabular} & \begin{tabular}{|l|} 
Radio- \\
logical \\
Patient
\end{tabular} & $\begin{array}{c}\text { Depth } \\
\text { Available distal space } \\
\text { Angulation } \\
\text { Root morphology } \\
\text { (curvature and num- } \\
\text { ber of roots) } \\
\text { Second molar rela- } \\
\text { tionship } \\
\text { Age } \\
\text { BMI }\end{array}$ & 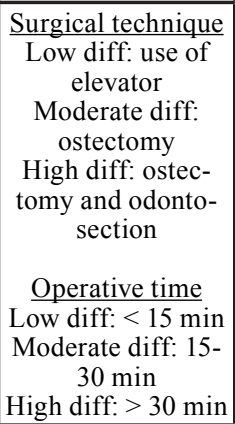 & $\begin{array}{l}1 \text { indepen- } \\
\text { dent eva- } \\
\text { luator }\end{array}$ & $\begin{array}{l}1 \text { senior } \\
\text { surgeon }\end{array}$ & $\begin{array}{c}\text { Se: } 93.1 \% \\
\text { Sp: } 87.9 \% \\
\text { Precision: } \\
90.4 \% \\
\text { PPV: } 87.1 \% \\
\text { NPV: } 93.6 \%\end{array}$ & $2++$ \\
\hline $\begin{array}{l}\text { Di- } \\
\text { niz-Fre- } \\
\text { itas } \text { et } \\
\text { al. } 2006 \\
(21)\end{array}$ & $\begin{array}{l}73 / \\
105\end{array}$ & $\begin{array}{l}\text { Pre-exis- } \\
\text { tent index } \\
\text { / scale } \\
\text { evaluation }\end{array}$ & Pederson & $\begin{array}{l}\text { Radio- } \\
\text { logical }\end{array}$ & $\begin{array}{c}\text { Depth } \\
\text { Available distal space } \\
\text { Angulation }\end{array}$ & $\begin{array}{l}\text { Modified Parant } \\
\text { scale* } \\
\text { Operative time }\end{array}$ & $\mathrm{NC}$ & \begin{tabular}{|c|}
5 resi- \\
dents of \\
2nd year \\
of Master \\
of Sur- \\
gery
\end{tabular} & $\begin{array}{c}\text { Between } \\
\text { scales: } \\
\mathrm{Se}=23.8 \% \\
\mathrm{Sp}=76.2 \% \\
\\
\text { Operative } \\
\text { time: } \\
\text { Pederson: } \\
p=0.055 \\
\text { Modified } \\
\text { Parant: } \\
p=0.000\end{array}$ & $2+$ \\
\hline
\end{tabular}


Table 1 cont.: Main characteristics of the studies included.

\begin{tabular}{|c|c|c|c|c|c|c|c|c|c|c|}
\hline $\begin{array}{c}\text { Gar- } \\
\text { cía-Gar- } \\
\text { cía } \text { et } \\
\text { al. } 2000 \\
(19)\end{array}$ & $\begin{array}{l}-/ \\
166\end{array}$ & $\begin{array}{l}\text { Pre-exis- } \\
\text { tent index } \\
\text { / scale } \\
\text { evaluation }\end{array}$ & \begin{tabular}{|l} 
Pell \& \\
Gregory
\end{tabular} & $\begin{array}{l}\text { Radio- } \\
\text { logical }\end{array}$ & \begin{tabular}{|c|} 
Depth \\
Available distal space
\end{tabular} & $\begin{array}{l}\text { Modified Parant } \\
\text { scale* }\end{array}$ & $\mathrm{NC}$ & 1 surgeon & $\begin{array}{c}\text { Depth: } \\
\mathrm{Se}=15 \% \\
\mathrm{Sp}=88 \% \\
\\
\text { Available } \\
\text { distal space: } \\
\mathrm{Se}=50 \% \\
\mathrm{Sp}=62 \%\end{array}$ & $2+$ \\
\hline \multirow[t]{2}{*}{$\begin{array}{l}\text { Roy } e t \\
\text { al. 2015 } \\
\text { (24) }\end{array}$} & \multirow[t]{2}{*}{$\begin{array}{c}100 / \\
100\end{array}$} & \multirow[t]{2}{*}{$\begin{array}{l}\text { New index } \\
\text { / scale de- } \\
\text { velopment }\end{array}$} & $\begin{array}{l}\text { New } \\
\text { Index }\end{array}$ & $\begin{array}{l}\text { Radio- } \\
\text { logical } \\
\text { Patient }\end{array}$ & \begin{tabular}{|c|} 
Depth \\
Available distal space \\
Angulation \\
Root width \\
Root curvature \\
Distance to the eleva- \\
tor point \\
Mouth opening \\
Tongue size \\
External oblique crest \\
angle \\
Cheek flexibility \\
\end{tabular} & \multirow[t]{2}{*}{ Operative time } & \multirow[t]{2}{*}{$\mathrm{NC}$} & \multirow[t]{2}{*}{$\mathrm{NC}$} & $\begin{array}{c}\text { Agreement } \\
(\text { kappa })=89 \%\end{array}$ & \multirow[t]{2}{*}{$2+$} \\
\hline & & & Pederson & $\begin{array}{l}\text { Radio- } \\
\text { logical }\end{array}$ & \begin{tabular}{|c|} 
Depth \\
Available distal space \\
Angulation
\end{tabular} & & & & $\begin{array}{c}\text { Agree- } \\
\text { ment (ka- } \\
\text { ppa) }=66.5 \%\end{array}$ & \\
\hline $\begin{array}{c}\text { Sainz de } \\
\text { Baranda } \\
\text { et al. } \\
2019(31)\end{array}$ & $\begin{array}{c}118 / \\
118\end{array}$ & $\begin{array}{l}\text { Pre-exis- } \\
\text { tent index } \\
\text { / scale } \\
\text { evaluation }\end{array}$ & Pederson & $\begin{array}{l}\text { Radio- } \\
\text { logical }\end{array}$ & \begin{tabular}{|c|} 
Depth \\
Angulation \\
Available distal space
\end{tabular} & Operative time & $\mathrm{NC}$ & 1 surgeon & $p<0.001$ & $2+$ \\
\hline $\begin{array}{c}\text { Sam- } \\
\text { martino } \\
\text { et al. } \\
2017(26)\end{array}$ & $\begin{array}{c}200 / \\
200\end{array}$ & $\begin{array}{l}\text { New index } \\
\text { / scale de- } \\
\text { velopment }\end{array}$ & $\begin{array}{l}\text { New } \\
\text { index }\end{array}$ & $\begin{array}{l}\text { Radio- } \\
\text { logical }\end{array}$ & \begin{tabular}{|c|} 
Depth \\
Available distal space \\
Angulation \\
Bone density \\
Relationship with IAN \\
Buccolingual position \\
Tooth morphology \\
(with or without al- \\
terations) \\
\end{tabular} & $\begin{array}{cc}\text { Postoperative dif- } \\
\text { ficulty reported by } \\
\text { the surgeon: } \\
-\quad \text { Simple } \\
- & \text { Moderately } \\
& \text { simple } \\
- & \text { Difficult } \\
- & \text { Extremely } \\
& \text { difficult }\end{array}$ & $\begin{array}{l}1 \text { experien- } \\
\text { ced surgeon }\end{array}$ & \begin{tabular}{|c|}
5 special- \\
ized sur- \\
geon with \\
similar \\
experien- \\
cie
\end{tabular} & Kappa $=73 \%$ & $2+$ \\
\hline \multirow[t]{2}{*}{$\begin{array}{c}\text { Stacchi } \\
\text { et al. } \\
2018(29)\end{array}$} & \multirow[t]{2}{*}{$\begin{array}{l}124 / \\
124\end{array}$} & \multirow[t]{2}{*}{$\begin{array}{l}\text { Pre-exis- } \\
\text { tent index } \\
\text { / scale } \\
\text { evaluation }\end{array}$} & \begin{tabular}{|c|} 
Juodz- \\
balys \\
and \\
Daugela \\
scale \\
\end{tabular} & \multirow[t]{2}{*}{$\begin{array}{l}\text { Radio- } \\
\text { logical }\end{array}$} & \multirow[t]{2}{*}{$\begin{array}{l}\text { Mesiodistal position } \\
\text { Apicocoronal position } \\
\text { Buccolingual position }\end{array}$} & \multirow[t]{2}{*}{ Operative time } & \multirow[t]{2}{*}{$\begin{array}{l}1 \text { blinded } \\
\text { expert } \\
\text { surgeon }\end{array}$} & \multirow[t]{2}{*}{$\begin{array}{l}1 \text { expert } \\
\text { surgeon } \\
\text { in each } \\
\text { center }\end{array}$} & $\mathrm{R}^{2}=0.126$ & \multirow[t]{2}{*}{$2++$} \\
\hline & & & \begin{tabular}{|c|} 
Modified \\
Juodz- \\
balys \\
and \\
Daugela \\
scale \\
\end{tabular} & & & & & & $\mathrm{R}^{2}=0.204$ & \\
\hline \multirow[t]{2}{*}{$\begin{array}{l}\text { Yuasa } e t \\
\text { al. 2002 } \\
\text { (20) }\end{array}$} & \multirow[t]{2}{*}{$\begin{array}{c}44 / \\
44\end{array}$} & \multirow[t]{2}{*}{$\begin{array}{l}\text { New index } \\
\text { / scale de- } \\
\text { velopment }\end{array}$} & $\begin{array}{l}\text { New } \\
\text { index }\end{array}$ & $\begin{array}{l}\text { Radio- } \\
\text { logical }\end{array}$ & \begin{tabular}{|c|} 
Depth \\
Available distal space \\
Root width \\
\end{tabular} & \multirow[t]{2}{*}{$\begin{array}{c}\text { Operative time } \\
\text { Surgeon-reported } \\
\text { difficulty }\end{array}$} & \multirow[t]{2}{*}{$\begin{array}{l}1 \text { radiolo- } \\
\text { gist }\end{array}$} & \multirow{2}{*}{\begin{tabular}{|c} 
Maxil- \\
lofacial \\
surgeons \\
with at \\
least 5 \\
years' \\
experi- \\
ence
\end{tabular}} & $\begin{array}{l}\mathrm{Se}=85 \% \\
\mathrm{Sp}=92 \%\end{array}$ & \multirow[t]{2}{*}{$2++$} \\
\hline & & & Pederson & $\begin{array}{l}\text { Radio- } \\
\text { logical }\end{array}$ & \begin{tabular}{c|} 
Depth \\
Angulation \\
Available distal space
\end{tabular} & & & & $\begin{array}{l}\mathrm{Se}=50 \% \\
\mathrm{Sp}=92 \%\end{array}$ & \\
\hline \multirow[t]{2}{*}{$\begin{array}{l}\text { Zhang et } \\
\text { al. 2019 } \\
\text { (30) }\end{array}$} & \multirow[t]{2}{*}{$\begin{array}{c}203 / \\
203\end{array}$} & \multirow[t]{2}{*}{$\begin{array}{l}\text { New index } \\
\text { / scale de- } \\
\text { velopment }\end{array}$} & $\begin{array}{l}\text { New } \\
\text { index }\end{array}$ & $\begin{array}{l}\text { Radio- } \\
\text { logical } \\
\text { Patient }\end{array}$ & \begin{tabular}{|c|} 
Depth \\
Angulation \\
Root shape \\
Number of roots \\
Relationship with IAN \\
Age \\
\end{tabular} & \multirow[t]{2}{*}{ Operative time } & \multirow[t]{2}{*}{$\begin{array}{l}2 \text { senior } \\
\text { residents }\end{array}$} & \multirow[t]{2}{*}{$\begin{array}{l}1 \text { surgeon } \\
\text { with } 25 \\
\text { years of } \\
\text { experi- } \\
\text { ence }\end{array}$} & Kappa $77.9 \%$ & \multirow[t]{2}{*}{$2++$} \\
\hline & & & Pederson & $\begin{array}{l}\text { Radio- } \\
\text { logical }\end{array}$ & \begin{tabular}{|c|} 
Depth \\
Available distal space \\
Angulation
\end{tabular} & & & & Карра $65.3 \%$ & \\
\hline
\end{tabular}

All of them are prospective cohort studies that have assessed difficulty of lower impacted 3M removal and have measured radiological variables from panoramic radiographs. *Modified Parant scale from García-García et al. 2000: I: conventional extraction; II: ostectomy; III: ostectomy and crown sectioning; IV: complex extraction with root sectioning. Se: sensitivity, Sp: specificity, PPV: positive predictive value, NPV: negative predictive value. 
All studies showed an improvement on the prediction of the surgical difficulty when using the new indices/scales or the proposed modifications of the pre-existing ones in comparison with pre-existing indices. Only 1 study failed to demonstrate improvement of a new index with respect to the modified Parant scale (24).

Table 2 shows the proposal of a form for the assessment of surgical difficulty of upper or lower third molars removal based on the results of the present systematic review, which combines the scales/indices developed so far, and the individual variables or factors that have been related to an increase in surgical difficulty and which have been recorded in a recently published systematic review by the authors (6). However, some of these factors have not yet been demonstrated. For this reason, the authors have completed the evaluation form with some categories based on their clinical experience in the field of oral and maxillofacial surgery.
The form includes 3 groups of variables: features of the patient, and radiological and surgical features. It classifies each clinical case into 1 out of 3 categories of difficulty. The scoring was adopted from the 'Endodontic Case Difficulty Assessment Form and Guidelines'. Each item is scored with 1 point for low difficulty, 2 points for moderate difficulty and 5 points for high difficulty. If the sum of the points is less than 20, the case has a low difficulty, suggesting an easy surgical case, that is, a conventional extraction that can be performed by a supervised student or by a general dentist. If the sum is between 20 and 40 points, the case is classified as moderately difficult and should be operated by a dentist with training in oral surgery over 3 years or by a qualified generalist dentist with specific continuing education and over 5 years experience in oral surgery. In cases over 40 points, considered to be highly difficult, the surgical intervention should be reserved for senior surgeons with more than 10 years of experience.

Table 2: Proposed pre-surgical assessment form on surgical difficulty of upper or lower third molars removal.

\begin{tabular}{|c|c|c|c|c|}
\hline & LOW DIFFICULTY & MODERATE DIFFICULTY & HIGH DIFFICULTY \\
\hline \multicolumn{5}{|c|}{ PATIENTS' CHARACTERISTICS } \\
\hline \multicolumn{2}{|l|}{ Age } & $\square<25$ years & $\square 25-50$ years & $\square>50$ years \\
\hline \multicolumn{2}{|l|}{ BMI } & $\square<25$ & $\square 25-30$ & $\square>30$ \\
\hline \multicolumn{2}{|c|}{ Ethnic background } & $\square$ Caucasian & $\square$ Asian & $\square$ African \\
\hline \multicolumn{2}{|c|}{ Systemic disorders } & $\square$ ASA I & $\square$ ASA II & $\square$ ASA III y IV \\
\hline \multicolumn{2}{|l|}{ Anxiety level } & $\square$ Non anxious & $\square$ Anxious but cooperative & $\square$ Phobic, non cooperative \\
\hline \multicolumn{2}{|l|}{ Facial pattern } & $\square$ Dolichocephalic & $\square$ Mesocephalic & $\square$ Brachycefalic \\
\hline \multicolumn{2}{|c|}{ Mouth opening } & $\square>45 \mathrm{~mm}$ & $\square 35-45 \mathrm{~mm}$ & $\square<35 \mathrm{~mm}$ \\
\hline \multicolumn{5}{|c|}{ RADIOLOGICAL VARIABLES } \\
\hline \multicolumn{2}{|c|}{ Root morphology } & $\square$ Conical fused roots & $\square$ Multi-radicular ( $\geq 2$ roots) & $\begin{array}{l}\square \text { Germ } \\
\square \text { Bulbous roots } \\
\square \text { Dilacerated roots }\end{array}$ \\
\hline \multicolumn{2}{|c|}{ Available distal space } & $\square$ Pell \& Gregory I & $\square$ Pell \& Gregory II & $\square$ Pell \& Gregory III \\
\hline \multicolumn{2}{|l|}{ Depth } & $\square$ Pell \& Gregory A & $\square$ Pell \& Gregory B & $\square$ Pell \& Gregory C \\
\hline \multicolumn{2}{|l|}{ Angulation } & $\square$ Mesioangular & $\square$ Vertical & \begin{tabular}{|l} 
Horizontal \\
$\square$ Distoangular
\end{tabular} \\
\hline \multirow[b]{2}{*}{$\begin{array}{l}\text { Proximity to } \\
\text { anatomical } \\
\text { structures }\end{array}$} & \begin{tabular}{|l}
$\begin{array}{l}\text { Maxillary } \\
\text { sinus }\end{array}$ \\
\end{tabular} & $\begin{array}{l}\square \text { Apex without contact } \\
\text { with the cortex }\end{array}$ & $\square$ Apex in contact with the cortex & $\begin{array}{l}\square \text { Apex overlapping or } \\
\text { exceeding the cortex }\end{array}$ \\
\hline & $\begin{array}{l}\text { Inferior al- } \\
\text { veolar nerve }\end{array}$ & $\begin{array}{l}\square \text { Apex away from the } \\
\text { upper cortex of the lower } \\
\text { dental canal }\end{array}$ & $\begin{array}{l}\square \text { Apex overlapping the upper cortex } \\
\square \text { Darkening of the roots }\end{array}$ & $\begin{array}{l}\square \text { Overlay/loss of both cortices } \\
\square \text { Narrowing of the duct } \\
\square \text { Deviation of the duct } \\
\square \text { Bent apices }\end{array}$ \\
\hline \multicolumn{2}{|c|}{ Second molar relationship } & $\square$ Absent & $\begin{array}{l}\square \text { The tooth contacts the crown of } \\
\text { the 2nd molar }\end{array}$ & $\begin{array}{l}\square \text { The tooth contacts the crown } \\
\text { and/or root of the 2nd molar }\end{array}$ \\
\hline \multirow{2}{*}{\multicolumn{2}{|c|}{$\begin{array}{l}\text { Periodontal space } \\
\text { SURGICAL VARIABLES }\end{array}$}} & $\square$ Radiolucent & $\square$ Mixed & $\square$ Radio-opaque \\
\hline & & & & \\
\hline \multicolumn{2}{|l|}{ Anesthesia } & $\square$ No history of problems & $\square$ Vasoconstrictor intolerance & $\square$ Previous anesthetic failure \\
\hline \multicolumn{2}{|c|}{ Degree of impaction } & $\square$ Erupted & $\begin{array}{l}\square \text { Semierupted } \\
\square \text { Partial bony impactionl }\end{array}$ & $\square$ Intraosseous \\
\hline \multicolumn{2}{|c|}{ Surgical technique } & \begin{tabular}{|l|} 
Conventional extraction \\
with elevators and forceps \\
\end{tabular} & $\square$ Need of ostectomy & $\begin{array}{l}\square \text { Ostectomy and tooth } \\
\text { sectioning }\end{array}$ \\
\hline \multicolumn{2}{|c|}{$\begin{array}{l}\text { Presence of associated } \\
\text { lesions }\end{array}$} & $\begin{array}{l}\text { Absence of lesions } \\
\text { Thickened follicle } \\
\end{array}$ & $\square$ Associated lesion $<10 \mathrm{~mm}$ & $\square$ Associated lesion $>10 \mathrm{~mm}$ \\
\hline \multicolumn{2}{|c|}{ Surgeon's experience } & $\begin{array}{l}\square \text { Senior dentist with oral } \\
\text { surgery experience }>10 \\
\text { years }\end{array}$ & $\begin{array}{l}\square \text { Dentist with Master or regulated } \\
\text { training in oral surgery }>3 \text { years } \\
\square \text { General dentist with specific continu- } \\
\text { ing education in oral surgery }>5 \text { years }\end{array}$ & $\begin{array}{l}\square \text { General dentist } \\
\square \text { Supervised student }\end{array}$ \\
\hline
\end{tabular}




\section{Discussion}

This study aimed to collect pre-existing scales assessing the difficulty of third molars in order to design a difficulty assessment form to help professionals and to be used in an educational setting. The importance of having indices/scales that indicate the degree of surgical difficulty lies in a correct treatment planning to avoid underestimation of the difficulty and to minimize the number of intra- and post-operative complications (29). Some scales such as those of Pell and Gregory, Winter and Pederson are widely used although several studies have shown that they poorly predict surgical difficulty $(16,18)$. Juodzbalys and Daugela (29) carried out a literature review and designed an index/scale based on anatomical and radiological factors. This classification relates the $3 \mathrm{M}$ to adjacent structures such as the mandibular ramus, the second molar, the alveolar ridge, the mandibular canal, and the spatial position of the tooth. Another study published by Manuel et al. (30) shows a proforma for the collection of clinical history data in order to be used by residents of an oral and maxillofacial surgery service in India. The benefits of a good history are the early evaluation of difficulty and possible intra- and post-operative complications, among others. However, until now there is no specific tool to evaluate the surgical difficulty of $3 \mathrm{M}$ in a multidimensional way, as the one presented in this paper.

The results obtained in the present systematic review show that most of the existing indices/scales include radiological variables collected from panoramic radiographs, and only some contain variables or characteristics of the patient himself, such as age or BMI, among others. However, taking into account the results of a recently published systematic review (6), these scales are not aligned with the factors that have been shown to influence the increase in surgical difficulty. These are divided into three blocks: patient characteristics, radiological factors and surgical factors.

Surgical factors are usually treated separately from other factors. There are indices/scales that assess difficulty only by the type of surgical technique, such as the modified Parant scale (31). However, none of these refers to the surgeon's experience. The measurement of experience is a controversial issue. There are studies that refer to the number of years worked after completion of training (32) but some of those included in this review cite the senior category without explaining the number of years of experience $(23,25,26)$. In this line, a study published by Ashton-James et al. (33) determines experience in terms of the number of $3 \mathrm{M}$ extractions performed throughout the professional career. Although few, some studies have linked the surgeon's experience with post-operative complications and morbidity and have found more complications in less experienced professionals $(34,35)$ or non-specialized generalists $(35,36)$, as well as greater post-operative morbidity when the procedure is performed by generalists (35).

In the field of oral and maxillofacial surgery there are no studies to assess the learning curve of the extraction of impacted 3M. The learning curve is the time and/or number of surgical interventions that a novice surgeon needs to be able to perform a procedure independently and with a good result, thus becoming a competent surgeon (37). Therefore, during this training period until the surgeon acquires the necessary skills, the risk of morbidity and complications is higher (38).

However, although the more experienced surgeons have fewer post-operative complications, the less experienced ones may also have a low number of complications, provided that their learning curve is good and progressive (39). In the area of endoscopic surgery, $\mathrm{Qu}$ et al. (40) studied the performance of surgery for an endoscopic thyroidectomy with an intra-oral approach and concluded that this competence was acquired after 20 cases, when a significant reduction in operative time was observed. In their study they detail some of the more challenging surgical steps and therefore recommend that a novice surgeon initially imitate and practice under the close guidance of an experienced supervisor. Unfortunately, the number of interventions required to master or be competent at extracting $3 \mathrm{M}$ is unknown as this issue has not been studied in our field. In addition, it should be noted that individual learning will depend on the surgeon's own manual skill and knowledge of anatomy or technique. Usually, as the clinician acquires skills, the difficulty of the cases increases, which can have a temporary negative impact both in complication rates and operative time (37).

In our opinion, the learning curve on difficulty assessment has to be developed also during the first years of clinical practice, both for generalists and for specialists in oral and maxillofacial surgery. Correctly predicting the difficulty of the impacted $3 \mathrm{M}$ removal is relevant in order to avoid iatrogeny in less expert surgeons and perform a progressive learning curve.

Therefore, the development of the present form for assessing the difficulty of surgical extraction of $3 \mathrm{Ms}$ based on the available scientific evidence and the clinical experience of the authors is an opportunity to improve the training of students and to guide recent graduates and even oral surgery specialists. It is intended to help reduce intra- and post-operative complications and to assist with referral to an experienced surgeon.

\section{Conclusions}

The existing indices/scales are mainly based on radiological variables that can be evaluated in a panoramic radiography. Very few authors introduce variables related to the patient's own clinical characteristics. The few scales that evaluate surgical variables only include 
the type of surgical technique. None of them values the surgeon's experience.

The use of a protocol designed to evaluate the difficulty of $3 \mathrm{Ms}$ removal that includes patient-specific, radiological and surgical variables can facilitate treatment planning, help the professional foresee possible complications and decide whether to refer the patient to a specialist with proven knowledge and experience.

\section{References}

1. Gbotolorun OM, Arotiba GT, Ladeinde AL. Assessment of factors associated with surgical difficulty in impacted mandibular third molar extraction. J Oral Maxillofac Surg. 2007;65:1977-83.

2. Benediktsdóttir IS, Wenzel A, Petersen JK, Hintze H. Mandibular third molar removal: Risk indicators for extended operation time, postoperative pain, and complications. Oral Surg Oral Med Oral Pathol Oral Radiol Endod. 2004;97:438-46.

3. Renton T, Smeeton N, McGurk M. Factors predictive of difficulty of mandibular third molar surgery. Br Dent J. 2001;190:607-10.

4. Komerik N, Muglali M, Tas B, Selcuk U. Difficulty of impacted mandibular third molar tooth removal: Predictive ability of senior surgeons and residents. J Oral Maxillofac Surg. 2014;72:1062-6.

5. Ferrús-Torres E, Gargallo-Albiol J, Berini-Aytés L, Gay-Escoda C. Diagnostic predictability of digital versus conventional panoramic radiographs in the presurgical evaluation of impacted mandibular third molars. Int J Oral Maxillofac Surg. 2009;38:1184-7.

6. Sánchez-Torres A, Soler-Capdevila J, Ustrell-Barral M, Gay-Escoda C. Patient, radiological, and operative factors associated with surgical difficulty in the extraction of third molars: A systematic review. Int J Oral Maxillofac Surg. 2020;49:655-65.

7. Moher D, Liberati A, Tetzlaff J, Altman DG; PRISMA Group. Preferred reporting items for systematic reviews and meta-analyses: the PRISMA statement. Int J Surg. 2010;8:336-41.

8. Harbour R, Miller J. A new system for grading recommendations in evidence based guidelines. BMJ. 2001;323:334-6.

9. Kim JY, Yong HS, Park KH, Huh JK. Modified difficult index adding extremely difficult for fully impacted mandibular third molar extraction. J Korean Assoc Oral Maxillofac Surg. 2019;45:309-15.

10. Upadhyaya C, Chaurasia NK, Neupane I, Srivastava S. Incidence and pattern of impaction of mandibular third molars: a single institutional experience in Nepal. Kathmandu Univ Med J. 2017;15:67-70. 11. de Andrade PF, Silva JNN, Sotto-Maior BS, Ribeiro CG, Devito KL, Assis NMSP. Three-dimensional analysis of impacted maxillary third molars: A cone-beam computed tomographic study of the position and depth of impaction. Imaging Sci Dent. 2017;47:149-55.

12. Mahdey HM, Arora S, Wei M. Prevalence and difficulty index associated with the 3(rd) mandibular molar impaction among malaysian ethnicities: a clinico-radiographic study. J Clin Diagn Res. 2015:9:65-8

13. Lee JY, Do HS, Lim JH, Jang HS, Rim JS, Kwon JJ, et al. Correlation of antibiotic prophylaxis and difficulty of extraction with postoperative inflammatory complications in the lower third molar surgery. Br J Oral Maxillofac Surg. 2014;52:54-7.

14. Jerjes W, El-Maaytah M, Swinson B, Upile T, Thompson G, Gittelmon $\mathrm{S}$, et al. Inferior alveolar nerve injury and surgical difficulty prediction in third molar surgery: the role of dental panoramic tomography. J Clin Dent. 2006;17:122-30.

15. Jerjes W, Upile T, Kafas P, Abbas S, Rob J, McCarthy E, et al. Third molar surgery: the patient's and the clinician's perspective. Int Arch Med. 2009;2:32.

16. García AG, Sampedro FG, Rey JG, Vila PG, Martin MS. PellGregory classification is unreliable as a predictor of difficulty in extracting impacted lower third molars. Br J Oral Maxillofac Surg. 2000;38:585-7.

17. Yuasa H, Kawai T, Sugiura M. Classification of surgical difficulty in extracting impacted third molars. Br J Oral Maxillofac Surg. 2002;40:26-31
18. Diniz-Freitas M, Lago-Méndez L, Gude-Sampedro F, SomozaMartin JM, Gándara-Rey JM, García-García A. Pederson scale fails to predict how difficult it will be to extract lower third molars. Br J Oral Maxillofac Surg. 2007;45:23-6.

19. Akadiri OA, Fasola AO, Arotiba JT. Evaluation of Pederson index as an instrument for predicting difficulty of third molar surgical extraction. Niger Postgrad Med J. 2009;16:105-8.

20. Barreiro-Torres J, Diniz-Freitas M, Lago-Méndez L, GudeSampedro F, Gándara-Rey JM, García-García A. Evaluation of the surgical difficulty in lower third molar extraction. Med Oral Patol Oral Cir Bucal. 2010;15:869-74.

21. Roy I, Baliga SD, Louis A, et al. Importance of clinical and radiological parameters in assessment of surgical difficulty in removal of impacted mandibular 3rd molars: a new index. J Maxillofac Oral Surg. 2015;14:745-9.

22. Conti M, Valente A, Catelani M, Rao S. Assessment of difficulty in third impacted mandibular molar surgery: the Conti Scale. Minerva Stomatol. 2015;64:177-88.

23. Sammartino G, Gasparro R, Marenzi G, Trosino O, Mariniello M, Riccitiello F. Extraction of mandibular third molars: proposal of a new scale of difficulty. Br J Oral Maxillofac Surg. 2017;55:952-7.

24. Al-Samman AA. Evaluation of Kharma scale as a predictor of lower third molar extraction difficulty. Med Oral Patol Oral Cir Bucal. 2017;22:796-9.

25. de Carvalho RWF, Vasconcelos BC. Pernambuco index: predictability of the complexity of surgery for impacted lower third molars. Int J Oral Maxillofac Surg. 2018;47:234-40.

26. Stacchi C, Daugela P, Berton F, Lombardi T, Andriulionis T, Perinetti G, et al. A classification for assessing surgical difficulty in the extraction of mandibular impacted third molars: Description and clinical validation. Quintessence Int. 2018;49:745-53.

27. Zhang X, Wang L, Gao Z, Li J, Shan Z. Development of a New Index to assess the difficulty level of surgical removal of impacted mandibular third molars in an asian population. J Oral Maxillofac Surg. 2019;77:1-8.

28. Sainz de Baranda B, Silvestre FJ, Silvestre-Rangil J. Relationship between surgical difficulty of third molar extraction under local anesthesia and the postoperative evolution of clinical and blood parameters. J Oral Maxillofac Surg. 2019;77:1337-45.

29. Juodzbalys G, Daugela P. Mandibular third molar impaction: review of literature and a proposal of a classification. J Oral Maxillofac Res. 2013;4:1

30. Manuel S, Surej Kumar LK, Varghese MP. A comprehensive proforma for evaluation of mandibular third molar impactions. J Maxillofac Oral Surg. 2014;13:378-85.

31. Garcia Garcia A, Gude Sampedro F, Gandara Rey J, Gallas Torreira M. Trismus and pain after removal of impacted lower third molars. J Oral Maxillofac Surg. 1997;55:1223-6.

32. Susarla SM, Dodson TB. Estimating third molar extraction difficulty: a comparison of subjective and objective factors. J Oral Maxillofac Surg. 2005;63:427-34.

33. Ashton-James CE, Forouzanfar T, Costa D. The contribution of patients' presurgery perceptions of surgeon attributes to the experience of trust and pain during third molar surgery. Pain Rep. 2019;4:754.

34. Sisk AL, Hammer WB, Shelton DW, Joy ED Jr. Complications following removal of impacted third molars: the role of the experience of the surgeon. J Oral Maxillofac Surg. 1986;44:855-9.

35. Jerjes W, El-Maaytah M, Swinson B, Banu B, Upile T, D'Sa S. Experience versus complication rate in third molar surgery. Head Face Med. 2006;2:14

36. Berge TI, Gilhuus-Moe OT. Per- and post-operative variables of mandibular third-molar surgery by four general practitioners and one oral surgeon. Acta Odontol Scand. 1993;51:389-97.

37. Subramonian K, Muir G. The 'learning curve' in surgery: what is it, how do we measure it and can we influence it? BJU Int. 2004;93:1173-4

38. Benzel EC, Orr RD. A steep learning curve is a good thing! Spine J. 2011;11:131-2 
39. Mufson RA. The influence of experience on complication rates. J Oral Maxillofac Surg. 1987;45:906.

40. Qu R, Wang J, Li J, Dong Z, Yang J, Liu D, et al. The learning curve for surgeons regarding endoscopic thyroidectomy via the oral-vestibular approach. Surg Laparosc Endosc Percutan Tech. 2018;28:380-4.

\section{Acknowledgements}

This study was conducted by the research group "Dental and Maxillofacial Pathology and Therapeutics" of the Bellvitge Biomedical Research Institute (IDIBELL).

We thank CERCA Programme / Generalitat de Catalunya for institutional support.

\section{Funding}

This research has not received any specific funding from public, commercial, or non-profit sector funding agencies.

\section{Conflict of interest}

The authors deny any conflict of interest related to this study.

\section{Authors contributions}

Prof. Dr. Gay-Escoda participated in the design of the study, the interpretation of the results and the correction of the manuscript.

Dr. Sánchez-Torres participated in the preparation of the study, in the analysis of the results and in the writing of the manuscript.

Dr. Borrás-Ferreres participated in the preparation of the study, in the analysis of the results and in the writing of the manuscript.

Prof. Dr. Valmaseda-Castellón participated in the interpretation of the results and in the correction of the manuscript. 